\title{
Neoadjuvant and Adjuvant Immunotherapy in Early-Stage Non-Small Cell Lung Cancer
}

\section{Nikhil Shukla \\ Nasser Hanna}

Department of Hematology/Oncology, Indiana University Melvin and Bren Simon Cancer Center, Indianapolis, IN, 46202, USA
Correspondence: Nikhil Shukla Department of Hematology/Oncology, Indiana University Melvin and Bren Simon Cancer Center, 535 Barnhill Drive, RT 473, Indianapolis, IN, 46202, USA

Email nashukla@iu.edu
Abstract: Surgery or concurrent chemoradiation are standard of care treatments for patients with localized and locally advanced non-small cell lung cancer (NSCLC). While resection and chemoradiation are potentially curative therapies for early-stage disease, relapse rates remain high. Adjuvant or neoadjuvant chemotherapy improves cure rates 5-15\% compared with surgery alone for patients with resectable disease. Immune checkpoint inhibitors (ICI) have heralded a new era for the treatment of advanced NSCLC with one-third of patients experiencing long-term survival. There is increasing interest in examining the role of ICI therapy in patients with early-stage NSCLC. Consolidation durvalumab after chemoradiation has become a part of standard of care for patients with inoperable, locally advanced disease. More recently, there is emerging evidence that neoadjuvant treatment with ICIs results in substantial rates of major pathologic response and pathologic complete response, and high rates of R0 resection with no significant delay in time to surgery. Furthermore, preliminary data show that adjuvant treatment with ICIs after adjuvant chemotherapy improves diseasefree survival and may play a critical role in reducing disease recurrence in patients with resectable disease. In this review, we discuss recently reported and ongoing studies that are designed to define the role of immunotherapy in patients with non-metastatic NSCLC.

Keywords: early-stage NSCLC, immunotherapy, adjuvant, neoadjuvant

\section{Background}

Lung cancer is the most common malignancy and a leading cause of cancer-related death worldwide. ${ }^{1}$ NSCLC accounts for approximately $80 \%$ of all lung cancer diagnoses and $50 \%$ of patients present with localized or locally advanced disease. ${ }^{2}$ While many patients with early-stage disease are cured with surgical resection or concurrent chemoradiation (CRT), survival decreases with increasing stage at presentation. ${ }^{3}$ Most patients who develop locally recurrent or metastatic disease after resection or CRT are incurable with salvage therapy. The current standard of care for patients with stage IB-IIB (and some with IIIA) NSCLC involves neoadjuvant or adjuvant chemotherapy in addition to surgical resection. The addition of chemotherapy to surgery increases cure rates by 5 $15 \%{ }^{4-7}$ Until recently, the standard of care treatment for patients with inoperable stage III NSCLC was CRT with long-term survival plateauing at $15-20 \%{ }^{8}$. Given the poor prognosis for many patients with stage I-III disease who relapse after surgery or CRT, there remains a great need to identify novel approaches to improve survival outcomes.

The advent of immune checkpoint inhibitor (ICI) therapy has revolutionized the treatment of patients with advanced NSCLC and generated unprecedented increases in survival outcomes. ICI monotherapy and in combination with chemotherapy has resulted in durable responses with manageable toxicity in a subset of patients. ${ }^{9-11}$ 
As a result, ICI therapy has moved to the first-line treatment setting in most patients with metastatic NSCLC without targetable mutations. Additionally, the evidence that ICI therapy can lead to durable responses in some patients has led to several clinical trials incorporating ICIs into the treatment of localized or locally advanced disease. This review will focus on the rationale and emerging clinical data for incorporating ICIs in the treatment of nonmetastatic NSCLC.

\section{Neoadjuvant Immunotherapy in Resectable NSCLC - Review of the Data}

Preoperative systemic therapy for patients with resectable NSCLC offers the advantage of reducing tumor size, thereby increasing the rate of R0 resections, and abrogating progressive disease by earlier treatment of micrometastases. There is also emerging evidence that major pathologic response (MPR) after neoadjuvant treatment may provide a surrogate for long-term survival. ${ }^{12,13}$ The benefit of neoadjuvant chemotherapy was demonstrated in a large meta-analysis of 15 trials which included 2385 patients with predominantly squamous cell carcinomas or adenocarcinomas. The analysis showed a $10 \%$ reduction in distant metastases at 5 years, $13 \%$ relative risk reduction of death, and 5\% improvement in 5-year overall survival (OS) (HR 0.87, 95\% CI 0.78-0.96; p=0.007) in patients who received neoadjuvant chemotherapy. ${ }^{7}$ However, preoperative chemotherapy may result in delayed surgery for some patients who require additional time to recover from treatment-related adverse events. Despite this limitation, patients are more likely to receive perioperative chemotherapy if it is delivered in the neo-adjuvant, rather than the adjuvant setting.

Neoadjuvant immunotherapy with ICIs is an emerging treatment modality for patients with resectable disease. ICI therapy has a more favorable safety profile than platinumbased chemotherapy and has the potential for robust and durable anti-tumor responses against micrometastatic disease. Additionally, there is evidence that changes in the tumor microenvironment that allow for immune tolerance and escape occurs in early-stage disease. ${ }^{14,15} \mathrm{~A}$ recent meta-analysis demonstrated that patients with resected tumors harboring higher levels of CD8+ cytotoxic $\mathrm{T}$ cells, CD20+ $\mathrm{B}$ cells, and CD 56/57 NK cells had improved disease-free survival (DFS) and OS.
Conversely, patients whose tumors harbored increased FoxP3 + T regulatory cells had worse OS. ${ }^{16}$

One of the first studies to demonstrate promise for neoadjuvant immunotherapy was a pilot study of neoadjuvant nivolumab in patients with stage I-IIIA NSCLC (AJCC version 7). ${ }^{17}$ Nivolumab was given for two doses every 2 weeks approximately 4 weeks prior to planned surgical resection. There were no treatment-related delays to surgical resection. MPR, defined by $90 \%$ or greater tumor necrosis in the surgical specimen, was reported in $45 \%$ of the patients. At 12 months post-resection, $80 \%$ of the patients were alive and recurrence free. Two patients had isolated disease recurrences that were treated with local therapy and remained free from disease for at least 12 months of follow-up. Only a single patient died from recurrent lung cancer. A correlative analysis found that patients with a higher number of sequence alterations in the primary tumor had higher rates of pathologic response. Furthermore, patients with MPR had an increased number of tumor-specific T-cells as evidenced by a higher total number of T-cells (intra-tumoral and peripheral) with a restricted number of clones.

Additional trials (Table 1) with ICIs are ongoing and some studies have reported interim efficacy results. MK3475-223 is a Phase I neoadjuvant study of pembrolizumab (200 mg every 3 weeks) in patients with stage I-II NSCLC (AJCC version 7). ${ }^{18}$ A total of 10 patients were enrolled in the dose escalation cohort and received 2 cycles of pembrolizumab followed by surgery 1 week after treatment. No DLTs were reported in the dose escalation cohort. Four of 10 patients $(40 \%$; 95\% CI $16.7-$ $68.8 \%$ ) achieved MPR. Interestingly, there was no correlation between pre-treatment tumor PD-L1 score and MPR. A phase Ib study of neoadjuvant treatment with the PD-1 inhibitor Sintilimab (200 mg every 3 weeks) in patients with stage IA-IIIB NSCLC (AJCC version 8) has reported promising outcomes. ${ }^{19}$ A total of 37 patients received 2 cycles of Sintilimab and underwent surgical resection. Key secondary endpoints were overall response rate (ORR), MPR (defined as $\geq 90 \%$ reduction in tumor volume), DFS, and OS. Fifteen patients (40.5\%; 95\% CI $24.8-$ $57.9 \%$ ) achieved MPR and 6 patients achieved pathologic complete response (CR). The Lung Cancer Mutation Consortium (LCMC3) study, a Phase II trial of neoadjuvant atezolizumab in patients with stage IB to select stage IIIB NSCLC (AJCC version 7) has reported interim efficacy results. ${ }^{20}$ The primary endpoint is MPR, defined as $\leq 10 \%$ residual tumor volume. Patients received 2 cycles of atezolizumab $1200 \mathrm{mg}$ every 3 weeks prior to surgery. 
Table I Select Trials of Neoadjuvant Immunotherapy in Resectable NSCLC

\begin{tabular}{|c|c|c|c|c|c|c|c|}
\hline $\begin{array}{l}\text { Trial NCT } \\
\text { Number }\end{array}$ & Trial Name & Phase & Stage & Treatment & $\begin{array}{l}\text { Primary } \\
\text { Endpoint }\end{array}$ & $\begin{array}{c}\text { MPR } \\
\text { (No. of Patients) }\end{array}$ & $\begin{array}{c}\text { PCR } \\
\text { (No. of Patients) }\end{array}$ \\
\hline NCT0225962I & & Phase 2 & IB-IIIA & Nivolumab & $\begin{array}{l}\text { Safety/ } \\
\text { Feasibility }\end{array}$ & $9 / 20$ (45\%) & $3 / 20(15 \%)$ \\
\hline NCT0292730I & LCMC3 & Phase 2 & IB-IIIB & Atezolizumab & MPR & $15 / 77$ (19.5\%) & $4 / 77$ (5\%) \\
\hline $\begin{array}{l}\text { ChiCTR-OIC } \\
-17013726\end{array}$ & & $\begin{array}{l}\text { Phase } \\
\text { IB }\end{array}$ & IB-IIIA & Sintilimab & $\begin{array}{l}\text { Adverse } \\
\text { events }\end{array}$ & I5/37 (40.5\%) & $6 / 37$ (16.2\%) \\
\hline NCT03I58I29 & NEOSTAR & Phase 2 & $|A-I I| A$ & $\begin{array}{c}\text { Nivolumab or nivolumab }+ \\
\text { ipilimumab }\end{array}$ & MPR & $10 / 34(29 \%)$ & $6 / 34(15 \%)$ \\
\hline NCT02938624 & MK3475-223 & Phase I & I-II & Pembrolizumab & Safety/MPR & $4 / 10$ (40\%) & Not reported \\
\hline NCT028I8920 & TOP I50I & Phase 2 & IB-IIIA & Pembrolizumab & $\begin{array}{c}\text { Surgical } \\
\text { feasibility rate }\end{array}$ & Ongoing & Ongoing \\
\hline NCT02572843 & SAKK I6/I4 & Phase 2 & IIIA & Durvalumab & EFS & Ongoing & Ongoing \\
\hline
\end{tabular}

Abbreviations: MPR, major pathologic response; PCR, pathologic complete response; EFS, event free survival.

A total of 84 patients completed surgical resection. Excluding 7 patients with EGFR or ALK mutations, MPR was seen in 15 of 77 patients (19\%; 95\% CI 11$30 \%)$. Furthermore, 38 of 77 (49\%) of patients had tumor regression of $\geq 50 \%$. Similar to the results from MK3475223, MPR rates did not correlate with tumor mutational burden (TMB) or tumor PD-L1 scores. The NEOSTAR study is a randomized phase II trial of neoadjuvant nivolumab, nivolumab plus ipilimumab, nivolumab plus chemotherapy, or nivolumab plus ipilimumab plus chemotherapy in patients with stage IA-IIIA NSCLC (AJCC version 7). The primary endpoint is MPR, defined as $\leq 10 \%$ residual tumor volume. Interim efficacy results from nivolumab $(3 \mathrm{mg} / \mathrm{kg}$ days $1,15,29)$ and nivolumab plus ipilimumab ( $1 \mathrm{mg} / \mathrm{kg}$ day 1$)$ cohorts have been reported. ${ }^{21} \mathrm{~A}$ total of 44 patients were randomized, 23 receiving nivolumab and 21 receiving nivolumab plus ipilimumab. Of the 37 patients who underwent tumor resection, MPR rate was 35\% (24\% for nivolumab and $50 \%$ for nivolumab plus ipilimumab). Two patients in the nivolumab cohort achieved radiographic $\mathrm{CR}$ compared to six patients in the nivolumab plus ipilimumab cohort. MPR and radiographic CR rates were higher in patients with tumor PD-L1 $>1 \%$ compared to PD-L1 $\leq 1 \%$.

Collectively, these interim efficacy results for neoadjuvant ICIs demonstrate manageable toxicity with a high percentage of patients achieving MPR. The correlation between MPR and long-term outcomes will be defined by the results of ongoing Phase 3 trials.

\section{Neoadjuvant Chemoimmunotherapy in Resectable NSCLC - Review of the Data}

Induction chemotherapy has demonstrated increased survival outcomes in patients with stage IIIA NSCLC. ${ }^{22-25}$ Given the efficacy and manageable toxicity with the addition of ICIs to chemotherapy in patients with metastatic NSCLC, there are several ongoing and completed studies evaluating the safety and efficacy of neoadjuvant chemoimmunotherapy in patients with resectable NSCLC (Table 2).

Three recently completed studies have reported efficacy outcomes in patients with stage IIIA NSCLC treated with neoadjuvant chemoimmunotherapy. SAKK 16/14 was a phase II trial in patients with stage IIIA (AJCC vesion 7) NSCLC treated with neoadjuvant cisplatin $\left(100 \mathrm{mg} / \mathrm{m}^{2}\right)$ and Docetaxel $\left(85 \mathrm{mg} / \mathrm{m}^{2}\right)$ every 3 weeks for 2 cycles followed by 2 cycles of durvalumab $750 \mathrm{mg}$ every 2 weeks. ${ }^{26}$ Durvalumab was then continued for 1 year postoperatively. The primary endpoint was event-free survival (EFS) at 1 year. Of 68 patients enrolled, 58 completed neoadjuvant sequential chemoimmunotherapy and 55 patients underwent surgical resection. EFS at 12 months was $73.3 \%$ (90\% CI $62.5-81.4)$ and median OS has not been reached. More recently, the results of the phase II NADIM trial, which evaluated the safety and efficacy of neoadjuvant chemotherapy plus nivolumab in patients with 
Table 2 Select Trials of Neoadjuvant Chemoimmunotherapy in Resectable NSCLC

\begin{tabular}{|c|c|c|c|c|c|c|c|}
\hline $\begin{array}{l}\text { Trial NCT } \\
\text { Number }\end{array}$ & Trial Name & Phase & Stage & Treatment & $\begin{array}{l}\text { Primary } \\
\text { Endpoint }\end{array}$ & $\begin{array}{l}\text { MPR (No. of } \\
\text { Patients) }\end{array}$ & $\begin{array}{l}\text { PCR (No. of } \\
\text { Patients) }\end{array}$ \\
\hline NCT0308I689 & NADIM & Phase 2 & IIIA & Nivolumab + carboplatin/paclitaxel & PFS & $34 / 4 \mid(83 \%)$ & $26 / 41(63 \%)$ \\
\hline NCT027I6038 & & Phase 2 & IB-IIIA & $\begin{array}{c}\text { Atezolizumab }+ \text { cabroplatin } / \mathrm{Nab}- \\
\text { paclitaxel }\end{array}$ & MPR & $7 / I I(63.6 \%)$ & $3 / 11(37.3 \%)$ \\
\hline NCT02572843 & SAKK $6 / 14$ & Phase 2 & IIIA & Durvalumab + cisplatin/docetaxel & EFS & $33 / 55(60.0 \%)$ & $10 / 55(18.2 \%)$ \\
\hline NCT02998528 & $\begin{array}{l}\text { Checkmate } \\
816\end{array}$ & Phase 3 & IB-IIIA & $\begin{array}{c}\text { Nivolumab }+ \text { ipilimumab or nivolumab }+ \\
\text { chemotherapy }\end{array}$ & EFS/PCR & Not reported & $24 \%$ \\
\hline NCT04304248 & NeoTPDOI & Phase 2 & IIIA-IIIB & $\begin{array}{c}\text { Toripalimab }+ \text { carboplatin/pemetrexed or } \\
\text { Nab-paclitaxel }\end{array}$ & MPR & $20 / 30(66.7 \%)$ & $15 / 30(50 \%)$ \\
\hline NCT03838I59 & NADIM II & Phase 2 & IIIA-IIIB & Nivolumab + carboplatin/paclitaxel & PCR & Ongoing & Ongoing \\
\hline NCT0387II53 & $\begin{array}{c}\text { HCRN } \\
\text { LUNI7-32I }\end{array}$ & Phase 2 & III & $\begin{array}{c}\text { Durvalumab }+ \text { carboplatin/paclitaxel }+ \\
\text { radiation }\end{array}$ & PCR & Ongoing & Ongoing \\
\hline NCT03456063 & IMpower030 & Phase 3 & II-IIIB & Atezolizumab + platinum chemotherapy & MPR and EFS & Ongoing & Ongoing \\
\hline NCT0406I590 & & Phase 2 & I-IIIA & Pembrolizumab + cisplatin/pemetrexed & $\begin{array}{l}\% \text { of patients with } \\
\text { TIICs }\end{array}$ & Ongoing & Ongoing \\
\hline
\end{tabular}

Abbreviations: MPR, major pathologic response; PCR, pathologic complete response; EFS, event free survival; PFS, progression-free survival; TIIC, tumor infiltrating immune cells.

stage IIIA disease (AJCC version 7) were reported. ${ }^{27}$ A total of 46 patients were enrolled and received neoadjuvant carboplatin (area under the curve $6 ; 6 \mathrm{mg} / \mathrm{mL} / \mathrm{min}$ ), pacilitaxel $\left(200 \mathrm{mg} / \mathrm{m}^{2}\right)$, and nivolumab $(360 \mathrm{mg})$ on day 1 of a 21-day cycle for 3 cycles prior to surgical resection. Post-operatively, patients received adjuvant nivolumab (240 mg every 2 weeks for 4 months followed by $480 \mathrm{mg}$ every 4 weeks for a total of 12 months). A total of 41 patients underwent surgery after neoadjuvant therapy. In the intention to treat (ITT) population, progressionfree survival (PFS) was $95.7 \%$ (95\% CI 83.7-98.9) at 12 months, $87.0 \%$ (95\% CI 73.3-93.9) at 18 months, and $77.1 \%(95 \%$ CI $59.9-87.7)$ at 24 months. In the ITT population, OS was $97.8 \%(95 \%$ CI $85.5-99.7)$ at 12 months, $93.5 \%(95 \% \mathrm{CI} 81.1-97.8)$ at 18 months, and $89.9 \%$ (95\% CI 74.5-96.2) at 24 months. Of the patients who underwent surgery, 34 achieved MPR of whom 26 had CR. Furthermore, neoadjuvant treatment did not lead to death or delay in surgery. The NeoTPD01 study was a phase II trial in patients with stage IIIA or stage IIIB NSCLC (AJCC version 8) treated neoadjuvantly with the PD-1 inhibitor toripalimab $(240 \mathrm{mg})$, carboplatin (area under the curve 5 ), pemetrexed $\left(500 \mathrm{mg} / \mathrm{m}^{2}\right.$ for adenocarcinoma), or nab-paclitaxel $\left(260 \mathrm{mg} / \mathrm{m}^{2}\right)$ every 3 weeks for 3 cycles. The primary endpoint was MPR and secondary endpoints included pathologic CR, R0 resection rate, disease-free survival, and safety. A total of 33 patients were enrolled and 30 patients underwent resection. The MPR rate was $66.7 \%(20 / 30), 50 \%(15 / 30)$ of patients achieved pathologic CR, and $96.7 \%$ (29/30) of patients

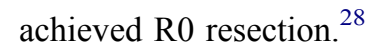

Results from the NADIM, SAKK 16/14, and NeoTPD01 trials suggest that the addition of ICIs to chemotherapy in the neoadjuvant setting is feasible in patients with stage IIIA disease. The majority of patients who received induction chemoimmunotherapy were able to undergo resection. Furthermore, although the data continues to mature, EFS and OS are particularly encouraging and seemingly superior to outcomes with standard of care treatment. ${ }^{3}$ Furthermore, results of the NADIM study suggest that chemoimmunotherapy may increase MPR rates compared to ICI monotherapy.

Concurrent chemoradiation plus ICI therapy in patients with resectable stage III disease is a logical approach to downstage nodal disease thereby increasing rates of $\mathrm{R} 0$ resection and increase rates of MPR. A phase I study of patients with stage IIIA disease treated with neoadjuvant concurrent CRT plus pembrolizumab was recently reported. ${ }^{29}$ Patients received cisplatin, etoposide, pembrolizumab $200 \mathrm{mg}$ every 3 weeks for 3 weeks and radiation 45 Gy in 25 fractions followed by 6 months of consolidation pembrolizumab. The primary objective was feasibility 
and safety, defined as grade $\leq 3$ pulmonary toxicity or any grade 4-5 nonhematologic toxicity. The study was stopped early after nine patients were enrolled due to two grade 5 events. Of the nine patients enrolled, six underwent complete resection with pathologic CR rate of $67 \%(4 / 6)$. The authors of the study note that the relationship between grade 5 events and the addition of pembrolizumab to CRT is unclear, but the study met the stopping rule for safety.

Efforts are also underway to evaluate the safety and efficacy of neoadjuvant chemoimmunotherapy in patients with earlier stage disease. Interim results from a single-arm phase II study of neoadjuvant atezolizumab (1200 mg every 3 weeks), carboplatin (area under the curve 6 every 3 weeks) and nab-paclitaxel $\left(100 \mathrm{mg} / \mathrm{m}^{2}\right.$ every week) for 4 cycles followed by resection in patients with stage IB-IIIA NSCLC (AJCC version 7) were reported. ${ }^{30}$ The primary endpoint is MPR, defined as $>90 \%$ decrease in viable tumor. Of 14 patients who underwent resection, 7 patients achieved MPR, including 3 patients with complete pathologic response (CR). Checkmate 816 is a multinational phase 3 study of patients with stage IB-stage IIIA NSCLC (AJCC version 7). ${ }^{31} \mathrm{~A}$ total of 642 patients are randomized 1:1:1 to receive nivolumab plus ipilimumab, nivolumab plus platinum doublet chemotherapy, or platinum doublet chemotherapy alone in the neoadjuvant setting. The primary endpoints are EFS and pathologic CR. Preliminary data released show that Checkmate 816 met its first primary endpoint with significantly improved pathologic CR in patients receiving nivolumab plus chemotherapy compared to chemotherapy alone ( $24 \%$ versus $2.2 \%$; odds ratio 13.94 [99\% CI 3.49-55.75]; $\mathrm{p}<0.0001) .{ }^{32}$

\section{Neoadjuvant Immunotherapy and Chemoimmunotherapy - Best Approaches}

Although ICI therapy has yet to be FDA approved in the neoadjuvant, reported efficacy data from trials has been promising and ICI therapy will likely play a major role in the treatment of patients with resectable NSCLC. Advantages to neoadjuvant immunotherapy or chemoimmunotherapy include the chance of MPR and improved DFS and OS. Data reported thus far calls into question the correlation between MPR with PD-L1 score or TMB. ${ }^{19,20}$ ICI therapy is less toxic than platinum doublet chemotherapy and neoadjuvant ICI monotherapy may be more appropriate for patients with contraindications to chemotherapy. For patients without contraindications to platinum doublet therapy, neoadjuvant chemoimmunotherapy has the benefit of higher rates of MPR and inducing responses in patients who may not benefit from ICI monotherapy. Neoadjuvant chemoimmunotherapy also the potential to augment downstaging of nodal disease particularly in patients with stage III disease, which has been previously associated with improved survival. ${ }^{12}$

However, not all patients will respond to neoadjuvant therapy and there is a risk of non-responding patients to become ineligible for curative resection due to progressive disease. Further analysis of biomarkers such as PD-L1, TMB, TILs, T-cell clonal profile that may predict response will need to be undertaken. Furthermore, patients with relative contraindications to ICI therapy may benefit from upfront surgical resection rather than risk toxicity and delayed resection. Patients with targetable mutations may also have limited benefit from neoadjuvant ICI therapy. Though data are very limited, among 45 patients with stage IB-IIIB who received neoadjuvant atezolizumab, 5 were found to have EGFR and ALK alterations. Data from three of these patients showed that one patient with an EGFR mutation was no longer resectable after neoadjuvant therapy. At the time of surgery, one patient with an EGFR mutation had $90 \%$ viable tumor and one patient with an ALK rearrangement had $60 \%$ viable tumor. ${ }^{33}$

Finally, while neoadjuvant concurrent CRT plus ICI therapy in patients with resectable stage III disease remains a logical treatment approach, preliminary Phase 1 data suggest the combination has high toxicity. ${ }^{28}$ Furthermore, pathologic CR rates were comparable to chemoimmunotherapy alone, suggesting that neoadjuvant radiation may not play a significant role in augmenting rates of MPR.

\section{Adjuvant Immunotherapy in Resectable NSCLC}

While neoadjuvant systemic therapy has the potential to reduce tumor size, increase R0 rates, and treat early micrometastatic disease, the primary goal of adjuvant therapy is to treat micrometastatic disease and prevent recurrence, while allowing for the primary tumor, which is the source of micrometastatic disease, to be removed as early as possible. Similar to neoadjuvant chemotherapy, efficacy of adjuvant chemotherapy in patients with stage IB-IIIA NSCLC has been demonstrated in multiple studies. ${ }^{4,6,34-36}$ The Lung Adjuvant Cisplatin Evaluation (LACE) meta- 
analysis included 4,584 patients from 5 cisplatin-based adjuvant trials and demonstrated a 5.4\% 5-year OS benefit for patients who received adjuvant chemotherapy. ${ }^{6}$ Given the small but consistent benefit from adjuvant chemotherapy in patients with early-stage NSCLC, there is increasing interest to determine if adjuvant immunotherapy can improve survival outcomes. The first reported adjuvant vaccine-based immunotherapy trial in NSCLC was a large, randomized Phase III trial of an immunotherapeutic targeting melanoma-associated antigen-A3 (MAGEA3), which enrolled 2,312 patients with stage IB-IIIA NSCLC to receive either the MAGE-A3 immunotherapeutic or placebo after resection. ${ }^{37}$ The trial results demonstrated no improvement in either DFS or OS in the MAGE-A3 arm.

Cellular therapy approaches have shown more promise in the adjuvant setting, with the most robust data from a randomized phase III trial of adjuvant chemoimmunotherapy with platinum doublet chemotherapy plus autologous activated killer T cells and dendritic cells compared to chemotherapy alone. ${ }^{38} \mathrm{~A}$ total of 103 patients who underwent surgical resection were randomized to the two arms. Patients with stage IIIB and stage IV with malignant pleural effusion as well as patients with mixed histology including small cell carcinoma were included. The study demonstrated impressive survival outcomes, with 2- and 5-year OS of 93.4\% (95\% CI 80.8-97.8) and 81.4\% (95\% CI $60.1-92.1)$ in the chemoimmunotherapy arm vs $66.0 \%$ (95\% CI 50.4-77.7) and $48.3 \%$ (95\% CI 31.5-63.3\%) in the chemotherapy arm, respectively. The HR $0.229(95 \%$ CI 0.093-0.564, p-0.0013) was statistically significant.
There is interest in incorporating ICI therapy following surgery in patients with resectable disease. ICI therapy is relatively well tolerated compared to cisplatin-based chemotherapy and offers the potential for durable, longlasting anti-tumor response. Furthermore, there is a preclinical rationale to incorporate ICI therapy in the adjuvant setting. Multiple studies have demonstrated that the trauma of surgical resection leads to an immunosuppressive environment that promotes tumor metastasis. ${ }^{39-42}$ In a surgical stress tumor mouse model, treatment with anti-PD-1 therapy restored CD8+ cytotoxic $\mathrm{T}$ cell activity. $^{43}$

Multiple phase II and phase III clinical trials (Table 3) are underway to actively explore these preclinical findings. IMpower010 is a randomized phase III trial in patients with stage IB-IIIA NSCLC (AJCC version 7) of adjuvant atezolizumab vs best supportive care after 4 cycles of cisplatin-based chemotherapy. ${ }^{44}$ Initial data at median 32.2 months of follow-up showed median disease-free survival (DFS) in patients with stage II-IIIA disease in the atezolizumab cohort versus best supportive care was 42.3 months versus 35.3 months (HR 0.79 , 95\% CI 0.64 $0.96, \mathrm{p}=0.025)$. The benefit of adjuvant atezolizumab was particularly pronounced in the PD-L1 $\geq 1 \%$ (HR $0.66,95 \%$ CI $0.49-0.97)$ and PD-L1 $\geq 50 \%$ (0.43 95\% CI 0.27-0.68) subgroups. The ANVIL portion of the ALCHEMIST trial, which randomizes patients with stage IB-stage IIIA NSCLC without EGFR or ALK mutations to receive 1 year of nivolumab or observation post-resection, has recently completed accrual. The primary endpoints are DFS and OS. Efficacy data has yet to be published.

Table 3 Select Trials of Adjuvant Immunotherapy in Resectable NSCLC

\begin{tabular}{|c|c|c|c|c|c|c|c|}
\hline $\begin{array}{l}\text { Trial NCT } \\
\text { Number }\end{array}$ & Trial Name & Phase & Stage & Treatment & Primary Endpoint & $\begin{array}{c}\text { Median DFS } \\
\text { (Months) }\end{array}$ & Trial Status \\
\hline NCT024867I8 & IMpower0I0 & Phase 3 & IB-IIIA & Atezolizumab & DFS & 42.3 & $\begin{array}{l}\text { Completed } \\
\text { accrual }\end{array}$ \\
\hline NCT02595944 & ANVIL & Phase 3 & IB-IIIA & Nivolumab & DFS and OS & Not reported & $\begin{array}{l}\text { Completed } \\
\text { Accrual }\end{array}$ \\
\hline NCT043I7534 & $\begin{array}{c}\text { BTCRC LUNI8- } \\
153\end{array}$ & Phase 2 & I & Pembrolizumab & DFS & Not reported & Ongoing \\
\hline NCT04585477 & & Phase 2 & I-III & Durvalumab & $\begin{array}{c}\text { Decrease in ctDNA } \\
\text { level }\end{array}$ & Not reported & Ongoing \\
\hline NCT02273375 & & Phase 3 & IB-IIIA & Durvalumab & DFS & Not reported & Ongoing \\
\hline NCT02504372 & PEARLS & Phase 3 & IB-IIIA & Pembrolizumab & DFS & Not reported & Ongoing \\
\hline
\end{tabular}

Abbreviations: DFS, disease free survival; ctDNA, circulating tumor DNA; OS, overall survival. 
Despite the promise of adjuvant ICI therapy, several questions remain unanswered. Key issues moving forward include the role of biomarkers in predicting DFS, if patients with earlier stage disease (node negative vs node positive) have the same degree of benefit from adjuvant ICI therapy, and duration of adjuvant ICI therapy necessary to achieve maximum survival benefit. Furthermore, the recently reported ADAURA trial, which evaluated adjuvant Osimertinib in patients with resected NSCLC with activating EGFR mutations, showed marked improvement in DFS in patients randomized to receive Osimertinib. ${ }^{45}$ The modest activity of ICI in patients with tumors that harbor EGFR mutations or ALK fusions mutations suggests that patients with some targetable mutations may not benefit from ICI therapy in the adjuvant setting. ${ }^{33}$ Additionally, nearly all studies of ICIs in the adjuvant setting are evaluating the role of ICI therapy after the completion of adjuvant chemotherapy. The role of adjuvant concomitant chemoimmunotherapy in improving DFS and OS is an important question yet to be addressed. One study is evaluating the efficacy of cisplatin-based chemotherapy plus atezolizumab in patients with stage I-IIIA NSCLC. The primary endpoint is percentage of patients with undetectable circulating tumor DNA (ctDNA) after adjuvant chemotherapy plus atezolizumab plus up to 13 cycles of atezolizumab (NCT04367311). Whether adjuvant ICI can overcome surgical immune suppression, prevent distant metastases, and improve survival in patients with resectable NSCLC remains to be seen.

\section{Consolidation Immunotherapy in Inoperable, Locally Advanced NSCLC}

Historically, the standard of care treatment for patients with inoperable stage III NSCLC steadily progressed from sequential to concurrent CRT with low rates of longterm survival. ${ }^{8}$ Unfortunately, many patients develop locally recurrent or metastatic disease, and most have micrometastatic disease at diagnosis. Both chemotherapy and radiation therapy are known to increase immunogenicity of tumors via increased neoantigen presentation with tumor cell death, inhibition of myeloid-derived suppressor cells, and increased ratio of cytotoxic and regulatory $\mathrm{T}$ cells in the peripheral circulation. ${ }^{46-50}$

The PACIFIC trial, a randomized phase III trial which compared treatment with consolidation durvalumab to placebo in over 700 patients, was the first to report significantly prolonged PFS and OS in patients who received consolidation durvalumab. ${ }^{51,52}$ Patients were randomized in a 2:1 fashion within 1-42 days after completion of CRT to receive durvalumab $(10 \mathrm{mg} / \mathrm{kg}$ every 2 weeks) for up to 1 year or placebo. The 12 and 18 month PFS was 55.9\% (95\% CI 51.0-60.4\%) and 44.2\% (95\% CI 37.7-50.5\%) for the durvalumab arm and 35.3\% (95\% CI $29.0-41.7 \%)$ and $27.0 \%$ (95\% CI $19.9-34.5 \%)$ for the placebo arm, respectively. The stratified HR for disease progression was $0.52 \quad(95 \%$ CI $0.42-0.65)$. Updated 5-year OS was $42.9 \%$ for the durvalumab arm and $33.4 \%$ for the placebo arm. The stratified HR for death was $0.72(95 \%$ CI $0.59-0.89){ }^{53}$ These results led to the FDA approval of durvalumab as consolidation therapy after CRT for patients with unresectable, locally advanced disease.

A similarly designed single-arm phase II study of consolidation pembrolizumab following CRT in patients with inoperable disease was recently reported. ${ }^{54} \mathrm{~A}$ total of 92 patients received pembrolizumab (200 mg every 3 weeks) for up to 1 year. After 32.2 months of follow-up, the median time to metastatic disease or death was 30.7 months (95\% CI 18.7 months to not reached). Median PFS was 18.7 months (12.4-33.8 months). The 1-, 2-, and 3-year OS survival estimates were $81.2 \%, 62.0 \%$, and $48.5 \%$, respectively. Recently, preliminary results from AFT-16 phase II trial of neoadjuvant and adjuvant atezolizumab and CRT for patients with unresectable stage III NSCLC was reported. The primary endpoint was disease control rate at 12 weeks and secondary endpoints included PFS, OS, overall response rate, and safety. A total of 64 patients were enrolled and at median followup of 24.1 months, median PFS was 23.7 months with OS at 18 months of $84 \%$ (95\% CI $75-94 \%)$ and median OS was not reached. ${ }^{55}$ A summary of completed and ongoing trials for patients with inoperable or unresectable disease is listed in Table 4.

Collectively, trials of consolidation ICI therapy have changed standard of care therapy for patients with unresectable stage III disease and have demonstrated that ICI therapy following CRT is safe and effective. However, several questions remained unanswered including role of ICI consolidation therapy in patients who are ineligible for chemotherapy and receive radiation therapy alone, optimal biomarkers to predict response to consolidation therapy, and whether patients with targetable mutations should receive consolidation therapy. 
Table 4 Select Trials of Concurrent/Consolidation Immunotherapy in Inoperable Locally Advanced NSCLC

\begin{tabular}{|c|c|c|c|c|c|c|c|}
\hline $\begin{array}{l}\text { Trial NCT } \\
\text { Number }\end{array}$ & Trial Name & Phase & Stage & Treatment & $\begin{array}{l}\text { Primary } \\
\text { Endpoint }\end{array}$ & $\begin{array}{l}\text { Median PFS } \\
\text { (Months) }\end{array}$ & $\begin{array}{c}\text { Median OS } \\
\text { (Months) }\end{array}$ \\
\hline NCT02I $2546 \mathrm{I}$ & PACIFIC & Phase 3 & III & Durvalumab & PFS and OS & 16.8 & 47.5 \\
\hline NCT02343952 & $\begin{array}{l}\text { HCRN LUNI4- } \\
\qquad 179\end{array}$ & Phase 2 & III & Pembrolizumab & TMDD & 18.7 & 35.8 \\
\hline NCT03।02242 & AFT-I6 & Phase 2 & III & Atezolizumab & $\begin{array}{l}\text { DCR at } 12 \\
\text { weeks }\end{array}$ & 23.7 & NR \\
\hline NCT035I997I & PACIFIC2 & Phase 3 & III & Durvalumab & PFS & Ongoing & Ongoing \\
\hline NCT0328532I & $\begin{array}{c}\text { BTCRC } \\
\text { LUNI6-08I }\end{array}$ & Phase 2 & III & $\begin{array}{c}\text { Nivolumab or nivolumab }+ \\
\text { ipilimumab }\end{array}$ & PFS & Ongoing & Ongoing \\
\hline NCT03663I66 & MCC-19704 & Phase $1 / 2$ & III & $\begin{array}{c}\text { CRT + ipilimumab followed by } \\
\text { nivolumab }\end{array}$ & Toxicity and PFS & Ongoing & Ongoing \\
\hline NCT045I3925 & $\begin{array}{l}\text { SKYSCRAPER- } \\
03\end{array}$ & Phase 3 & III & Atezolizumab + tiragolumab & PFS & Ongoing & Ongoing \\
\hline
\end{tabular}

Abbreviations: TMDD, time to metastatic disease or death; DCR, disease control rate; PFS, progression-free survival; OS, overall survival; CRT, chemoradiation.

\section{Concluding Thoughts}

Immunotherapy with ICIs has become a part of standard of care treatment for patients with advanced and locally advanced, unresectable NSCLC. Numerous studies are underway to determine the efficacy of ICI therapy in the treatment of early-stage NSCLC. ICIs given in the neoadjuvant setting have the potential to induce MPR, improve resectability, and diminish rates of distant metastases. Initial studies have reported MPR rates of $20-40 \%$ with no significant delay in surgery due to treatment toxicity. ${ }^{17-21}$ Furthermore, reported trials of neoadjuvant chemoimmunotherapy have shown impressive MPR and pathologic CR rates without major delays in time to surgery. ${ }^{26-28,30,32}$ Indeed, initial data from Checkmate 816 has shown that neoadjuvant chemoimmunotherapy increases rates of pathologic $\mathrm{CR}$ compared to chemotherapy alone. ${ }^{32}$ Whether MPR and pathologic CR translates into improved disease-free survival remains to be seen. While adjuvant immunotherapy with adoptive cellular therapy has shown promise in increasing disease-free and overall survival, the efficacy of ICI therapy after surgery is actively being investigated. Finally, consolidation ICI therapy has largely become standard of care practice for patients with inoperable stage III disease. Ongoing studies are evaluating the safety and efficacy of concurrent and/or neoadjuvant ICI therapy with CRT. Collectively, reported trials of checkpoint inhibitors in the neoadjuvant, adjuvant, and consolidation setting suggest that ICI therapy may augment cure rates and have a crucial role in the treatment of patients with early-stage NSCLC.

\section{Disclosure}

Nassar Hanna reports funding research from Merck, BMS, AstraZeneca, Genentech; Advisory board for "Beyond Spring" for work on the DSMB of a study; Royalties from serving as a writer for "UptoDate". The authors report no other conflicts of interest in this work.

\section{References}

1. Bray J, Ferlay J, Soerjomatram I, et al. Global cancer statistics 2018: GLOBOCAN estimates of incidence and mortality worldwide for 36 cancers in 185 countries. CA Cancer J Clin. 2018;68:394-424. doi:10.3322/caac. 21492

2. Ettinger DS, Akerley W, Borghaei H, et al. Non-small cell lung cancer. J Natl Compr Cancer Netw. 2012;10:1236-1271. doi:10.6004/ jncen.2012.0130

3. Goldstraw P, Chansky K, Crowley J, et al. The IASLC lung cancer staging project: proposals for revision of the TNM stage groupings in the forthcoming (eighth) edition of the TNM classification for lung cancer. $J$ Thorac Oncol. 2016;11:39-51. doi:10.1016/j.jtho.20 15.09.009

4. Douillard JY, Rosell R, De Lena M, et al. Adjuvant vinorelbine plus cisplatin versus observation in patients with completely resected stage IB-IIIA non-small-cell lung cancer (Adjuvant Navelbine International Trialist Association [ANITA]): a randomised controlled trial. Lancet Oncol. 2006;7:719-727. doi:10.1016/S1470-2045(06)70804-X

5. Winton T, Livingston R, Johnson D, et al. Vinorelbine plus cisplatin vs. observation in resected non-small-cell lung cancer. $N$ Engl J Med. 2005;352:2589-2597. doi:10.1056/NEJMoa043623

6. Pignon JP, Tribodet H, Scagliotti GV, et al. Lung adjuvant cisplatin evaluation: a pooled analysis by the LACE Collaborative Group. $J$ Clin Oncol. 2008;26:3552-3559. doi:10.1200/JCO.2007.13.9030 
7. NSCLC Meta-analysis Collaborative Group. Preoperative chemotherapy for non-small-cell lung cancer: a systematic review and meta-analysis of individual participant data. Lancet. 2014;383:1561-1571. doi:10.1016/S0140-6736(13)62159-5

8. Dillman DO, Herndon J, Seagren SL, et al. Improved survival in stage III non-small-cell lung cancer: seven-year follow-up of cancer and leukemia group B (CALGB) 8433 trial. J Natl Cancer Inst. 1996;88:1210-1215. doi:10.1093/jnci/88.17.1210

9. Reck M, Rodriguez-Abreu D, Robinson A, et al. Pembrolizumab versus chemotherapy for PD-L1-positive non-small-cell lung cancer N Engl J Med. 2016;375:1823-1833. doi:10.1056/NEJMoa1606774

10. Gandhi L, Rodriguez-Abreu D, Gadgeel S, et al. Pembrolizumab plus chemotherapy in metastatic non-small-cell lung cancer. $N$ Engl J Med. 2018;378:2078-2092. doi:10.1056/NEJMoa1801005

11. Paz-Ares L, Luft A, Vincente D, et al. Pembrolizumab plus chemotherapy for squamous non-small-cell lung cancer. $N$ Engl J Med. 2018;379:2040-2051. doi:10.1056/NEJMoa1810865

12. Hellmann MD, Chaft JE, William WN, et al. Pathological response after neoadjuvant chemotherapy in resectable non-small-cell lung cancers: proposal for the use of major pathological response as a surrogate endpoint. Lancet Oncol. 2014;15(1):e42-50. doi:10.1016/S1470-2045(13)70334-6

13. Pataer A, Kalhor N, Correa AM, et al. Histopathologic response criteria predict survival of patients with resected lung cancer after neoadjuvant chemotherapy. $J$ Thorac Oncol. 2012;7:825-832. doi:10.1097/JTO.0b013e318247504a

14. Lavin YT, Kobayashi S, Leader A, et al. Innate immune landscape in early lung adenocarcinoma by paired single-cell analyses. Cell. 2017;169:750-765. doi:10.1016/j.cell.2017.04.014

15. Schoenhals JE, Seyedin SN, Anderson C, et al. Uncovering the immune tumor microenvironment in non-small cell lung cancer to understand response rates to checkpoint blockade and radiation. Transl Lung Cancer Res. 2017;6:148-158. doi:10.21037/tlcr.2017.03.06

16. Tuminello S, Veluswamy R, Lieberman-Cribbin W, et al. Prognostic value of immune cells in the tumor microenvironment of early-stage lung cancer: a meta-analysis. Oncotarget. 2019;10:7142-7155. doi:10.18632/oncotarget.27392

17. Forde PM, Chaft JE, Smith KN, et al. Neoadjuvant PD-1 Blockade in Resectable Lung Cancer. N Engl J Med. 2018;378:1976-1986. doi:10.1056/NEJMoa1716078

18. Bar J, Urban D, Ofek E, et al. Neoadjuvant pembrolizumab (Pembro) for early stage non-small cell lung cancer (NSCLC): updated report of a phase I study, MK3475-223. J Clin Oncol. 2019;37:8534. doi:10.1200/JCO.2019.37.15_suppl.8534

19. Gao S, Li N, Gao S, et al. Neoadjuvant PD-1 inhibitor (Sintilimab) in NSCLC. $J$ Thorac Oncol. 2020;15:816-826. doi:10.1016/j. jtho.2020.01.017

20. Kwiatowski DJ, Rusch VW, Chaft JE. Neoadjuvant atezolizumab in resectable non-small cell lung cancer (NSCLC): interim analysis and biomarker data from a multicenter study (LCMC3). J Clin Oncol. 2019;37:8503. doi:10.1200/JCO.2019.37.15_suppl.8503

21. Cascone T, William WN, Weissferdt A, et al. Neoadjuvant nivolumab or nivolumab plus ipilimumab in operable non-small cell lung cancer: the Phase 2 randomized NEOSTAR trial. Nature. 2021;27:504-514.

22. Pass HI, Pogrebniak HW, Steinberg SM, Mulshine J, Minna J. Randomized trial of neoadjuvant therapy for lung cancer: interim analysis. Ann Thorac Surg. 1992;53:992-998. doi:10.1016/00034975(92)90373-C

23. Rosell R, Gomez-Codina J, Camps C, et al. A randomized trial comparing preoperative chemotherapy plus surgery with surgery alone in patients with non-small-cell lung cancer. $N$ Engl $\mathrm{J} \mathrm{Med}$. 1994;330:153-158. doi:10.1056/NEJM199401203300301

24. Roth JA, Fossella F, Komaki R, et al. A randomized trial comparing perioperative chemotherapy and surgery with surgery alone in resectable stage IIIA non-small-cell lung cancer. J Natl Cancer Inst. 1994;86:673-680. doi:10.1093/jnci/86.9.673
25. Depierre A, Milleron B, Moro-Sibilot D, et al. Preoperative chemotherapy followed by surgery compared with primary surgery in resectable stage I (except T1N0), II, and IIIa non-small-cell lung cancer. $J$ Clin Oncol. 2002;20:247-253. doi:10.1200/ JCO.2002.20.1.247

26. Rothschild S, Zippelius A, Eboulet EI, et al. SAKK 16/14: anti-PDL1 antibody durvalumab in addition to neoadjuvant chemotherapy in patients with stage IIIA(N2) non-small cell lung cancer (NSCLC)-A multicenter single-arm phase II trial. J Clin Oncol. 2020;38:suppl; abstr 9016. doi:10.1200/JCO.2020.38.15_suppl.9016

27. Provencio M, Nadal E, Insa A, et al. Neoadjuvant chemotherapy and nivolumab in resectable non-small-cell lung cancer (NADIM): an open-label, multicentre, single-arm, phase 2 trial. Lancet Oncol. 2020;21(11):1413-1422. doi:10.1016/S1470-2045(20)30453-8

28. Zhao Z, Chen S, Qi H, et al. Phase II trial of toripalimab plus chemotherapy as neoadjuvant treatment in resectable stage III non-small cell lung cancer (NeoTPD01 Study). J Clin Oncol. 2021;39(suppl 15):8541. doi:10.1200/JCO.2021.39.15_suppl.8541

29. Lemmon C, Gregory MM, Murthy SC, et al. A phase I safety and feasibility study of neoadjuvant chemoradiation plus pembrolizumab followed by consolidation pembrolizumab in resectable stage IIIA non-small cell lung cancer. J Clin Oncol. 2020;38:9009. doi:10.1200/ JCO.2020.38.15_suppl.9009

30. Shu CA, Grigg C, Chiuzan C, et al. Neoadjuvant atezolizumab + chemotherapy in resectable non-small cell lung cancer (NSCLC). $J \quad$ Clin Oncol. 2018;36:8532. doi:10.1200/JCO.2018.36.1 5_suppl.8532

31. Felip E, Brahmer J, Broderick S, et al. Checkmate 816: a phase 3 trial of neoadjuvant nivolumab plus ipilimumab or chemotherapy vs chemotherapy in early-stage NSCLC. J Thorac Oncol. 2018;13:S831S832. doi:10.1016/j.jtho.2018.08.1478

32. Forde PM, Spicer J, Lu S, et al. Nivolumab (NIVO) + platinum-doublet chemotherapy (chemo) vs chemo as neoadjuvant treatment (tx) for resectable (IB-IIIA) non-small cell lung cancer (NSCLC) in the phase 3 CheckMate 816 trial. In: Proceedings of the 112th Annual Meeting of the American Association for Cancer Research; April 10-15, 2021; Philadelphia (PA): AACR. Abstract CT003.

33. Rusch VW, Chaft JE, Johnson B, et al. Neoadjuvant atezolizumab in resectable non-small cell lung cancer (NSCLC): updated results from a multicenter study (LCMC3). J Thorac Oncol. 2018;13:S369. doi:10.1016/j.jtho.2018.08.346

34. Arriagada R, Bergman B, Dunant A, et al. Cisplatin-based adjuvant chemotherapy in patients with completely resected non-small-cell lung cancer. N Engl J Med. 2004;350:351-360.

35. Butts CA, Ding K, Seymour L, et al. Randomized phase III trial of vinorelbine plus cisplatin compared with observation in completely resected stage IB and II non-small-cell lung cancer: updated survival analysis of JBR-10. J Clin Oncol. 2010;28:29-34. doi:10.1200/ JCO.2009.24.0333

36. Scagliotti GV, Fossati R, Torri V, et al. Randomized study of adjuvant chemotherapy for completely resected stage I, II, or IIIA non-smallcell lung cancer. J Natl Cancer Inst. 2003;95:1453-1461. doi:10.1093/jnci/djg059

37. Vansteenkiste JF, Cho BC, Vanakesa T, et al. Efficacy of the MAGE-A3 cancer immunotherapeutic as adjuvant therapy in patients with resected MAGE-A3-positive non-small-cell lung cancer (MAGRIT): a randomised, double-blind, placebo-controlled, phase 3 trial. Lancet Oncol. 2016;17:822-835. doi:10.1016/S1470-2045(16) 00099-1

38. Kimura H, Matsui Y, Ishikawa A, et al. Randomized controlled phase III trial of adjuvant chemo-immunotherapy with activated killer $\mathrm{T}$ cells and dendritic cells in patients with resected primary lung cancer. Cancer Immunol Immunother. 2015;64:51-59. doi:10.1007/ s00262-014-1613-0 
39. Espi A, Arenas J, Garcia-Granero E, Marti E, Lledo S. Relationship of curative surgery on natural killer cell activity in colorectal cancer. Dis Colon Rectum. 1996;39:429-434. doi:10.1007/BF02054059

40. Coffey JC, Wang JH, Smith MJF, et al. Excisional surgery for cancer cure: therapy at a cost. Lancet Oncol. 2003;4:760-768. doi:10.1016/ S1470-2045(03)01282-8

41. Tai L, de Souza C, Bélanger S, et al. Preventing postoperative metastatic disease by inhibiting surgery-induced dysfunction in natural killer cells. Cancer Res. 2013;1:97-107. doi:10.1158/0008-5472. CAN-12-1993

42. Ananth A, Tai L, Lansdell C, et al. Surgical stress abrogates pre-existing protective $\mathrm{T}$ cell mediated anti-tumor immunity leading to postoperative cancer recurrence. PLoS One. 2016;11:1-19.

43. Sun Z, Mao A, Wang Y, et al. Treatment with anti-programmed cell death 1 (PD-1) antibody restored postoperative CD8 $+\mathrm{T}$ cell dysfunction by surgical stress. Biomed Pharmacother. 2017;89:1235-1241. doi:10.1016/j.biopha.2017.03.014

44. Wakelee H, Altorki NK, Zhou C, et al. IMpower010: primary results of a phase III global study of atezolizumab versus best supportive care after adjuvant chemotherapy in resected stage IB-IIIA non-small cell lung cancer (NSCLC). J Clin Oncol. 2021;39(suppl15):8500. doi:10.1200/JCO.2021.39.15_suppl.8500

45. Wu YL, Tsuboi M, He J, et al. Osimertinib in resected EGFRmutated non-small-cell lung cancer. $N$ Engl $J$ Med. 2020;383:1711-1723. doi:10.1056/NEJMoa2027071

46. Bracci L, Schiavoni G, Sistigu A. Immune-based mechanisms of cytotoxic chemotherapy: implications for the design of novel and rationale-based combined treatments against cancer. Cell Death Differ. 2014;21:15-25. doi:10.1038/cdd.2013.67

47. Wang Z, Till B, Gao Q. Chemotherapeutic agent-mediated elimination of myeloid-derived suppressor cells. Oncoimmunology. 2017;6: e1331807. doi:10.1080/2162402X.2017.1331807
48. Roselli M, Cereda V, Di Bari MG, et al. Effects of conventional therapeutic interventions on the number and function of regulatory $\mathrm{T}$ cells. Oncoimmunology. 2013;2:e27025. doi:10.4161/onci.27025

49. Tang $\mathrm{C}$, Wang $\mathrm{X}$, Soh $\mathrm{H}$, et al. Combining radiation and immunotherapy: a new systemic therapy for solid tumors? Cancer Immunol Res. 2014;2:831-838. doi:10.1158/2326-6066.CIR-14-0069

50. Deng L, Liang H, Burnette Bet al. Irradiation and anti-PD-L1 treatment synergistically promote antitumor immunity in mice. $J$ Clin Inves. 2014;124:687-695. doi:10.1172/JCI67313

51. Antonia SJ, Villegas A, Daniel D, et al. Durvalumab after chemoradiotherapy in stage III non-small-cell lung cancer. $N$ Engl J Med. 2017;377:1919-1929. doi:10.1056/NEJMoa1709937

52. Antonia SJ, Villegas A, Daniel D, et al. Overall survival with durvalumab after chemoradiotherapy in stage III NSCLC. $N$ Engl J Med. 2018;379:2342-2350.

53. Spigel DR, Faivre-Finn C, Gray JE, et al. Five-year survival outcomes with durvalumab after chemoradiotherapy in unresectable stage III NSCLC: an update from the PACIFIC trial. J Clin Oncol. 2021;39(suppl 15):8511. doi:10.1200/JCO.2021.39.15_suppl.8511

54. Durm GA, Jabbour SK, Althouse SK, et al. A phase 2 trial of consolidation pembrolizumab following concurrent chemoradiation for patients with unresectable stage III non-small cell lung cancer: Hoosier Cancer Research Network LUN 14-179. Cancer. 2020;126:4353-4361.

55. Ross HJ, Kozono DE, Urbanic JJ, et al. AFT-16: phase II trial of neoadjuvant and adjuvant atezolizumab and chemoradiation (CRT) for stage III non-small cell lung cancer (NSCLC). J Clin Oncol. 2021;39(suppl 15):8513. doi:10.1200/JCO.2021.39.15_suppl.8513
Lung Cancer: Targets and Therapy

\section{Publish your work in this journal}

Lung Cancer: Targets and Therapy is an international, peerreviewed, open access journal focusing on lung cancer research, identification of therapeutic targets and the optimal use of preventative and integrated treatment interventions to achieve improved outcomes, enhanced survival and quality of life for the cancer patient. Specific topics covered in the journal include: Epidemiology,

\section{Dovepress}

detection and screening; Cellular research and biomarkers; Identification of biotargets and agents with novel mechanisms of action; Optimal clinical use of existing anticancer agents, including combination therapies; Radiation and surgery; Palliative care; Patient adherence, quality of life, satisfaction; Health economic evaluations. 